\title{
REGINE KAHL
}

\section{Das Graduiertenkolleg 1427 „Nahrungsinhaltsstoffe als Signalgeber nukleärer Rezeptoren im Darm“}

Das Graduiertenkolleg 1427 „Nahrungsinhaltsstoffe als Signalgeber nukleärer Rezeptoren im Darm“ hat seine Arbeit im Wintersemester 2006/2007 aufgenommen. In den neun Projekten des Kollegs forschen elf von der Deutschen Forschungsgemeinschaft finanzierte Stipendiatinnen und Stipendiaten und zehn aus anderen Mitteln finanzierte Kollegiatinnen und Kollegiaten mit Studienabschlüssen in Biologie, Biochemie, Chemie und Ökotrophologie. Beteiligt sind Arbeitsgruppen der Mathematisch-Naturwissenschaftlichen und der Medizinischen Fakultät der Heinrich-Heine-Universität, des Instituts für Umweltmedizinische Forschung an der Heinrich-Heine-Universität (IUF), des Instituts für Arbeitsphysiologie an der Technischen Universität Dortmund (IfADo) und der Deutschen Sporthochschule Köln.

\section{Hintergrund}

Lebensmittelsicherheit ist ein zentrales Anliegen der Öffentlichkeit. Das Risikomanagement auf diesem Gebiet muss sich auf eine verlässliche wissenschaftliche Datenbasis zu den Wirkungen der Nahrung auf die menschliche Gesundheit stützen. Das Graduiertenkolleg 1427 verbindet dieses gesundheitspolitische Anliegen mit der Chance zu hochrangiger biomedizinischer Grundlagenforschung, die sich bei der Beschäftigung mit den zellulären und molekularen Angriffspunkten von Nahrungsinhaltsstoffen ergibt. Vor dem Hintergrund des aktuellen Wissensstandes über Zellzyklus, Proliferation und Zelltod zeichnet sich ab, dass bekannte Schadstoffe in der Nahrung, aber auch sekundäre Pflanzeninhaltsstoffe, denen eine Schutzwirkung gegen Krankheit und Altern zugeschrieben wird, weit differenziertere Wirkungsmechanismen aufweisen als früher vermutet. Der Fokus der Forschung hat sich in den letzten Jahren von schädigenden beziehungsweise schützenden Effekten am einzelnen Molekül auf Eingriffe in das hochkomplexe Netzwerk der zellulären Regulation verlagert. Man geht heute davon aus, dass sowohl chemische Stoffe, die als gesundheitsgefährdend gelten (wie polyzyklische aromatische Kohlenwasserstoffe, Dioxine, Nanopartikel oder Schwermetalle wie Cadmium), als auch sekundäre Pflanzeninhaltsstoffe, denen eine Schutzwirkung auf die menschliche Gesundheit zugeschrieben wird und die von vielen Menschen als Nahrungsergänzungsmittel prophylaktisch verzehrt werden (wie Vitamine und Antioxidantien), über die Bindung an nukleäre Rezeptoren auf die Genexpression einwirken und/oder mit Signaltransduktionsprozessen interferieren, die die Genexpression regulieren. Das Forschungsprogramm des Graduiertenkollegs ist so angelegt, 
dass einerseits Kenntnisse über die Wirkungsweise von Lebensmittelinhaltsstoffen generiert werden können, die bei der Risikoabschätzung und regulatorischen Bewertung unverzichtbar sind, und andererseits die untersuchten Lebensmittelinhaltsstoffe als Werkzeuge für die Aufklärung von Teilprozessen der zellulären Regulation genutzt werden können. Die Projekte konzentrieren sich auf das Erstkontaktgewebe Darm und seine Suszeptibilität für Nahrungsbestandteile und beziehen auch immunologische Fragestellungen mit ein.

\section{Didaktisches Konzept}

Die Studierenden durchlaufen eine strukturierte Doktorandenausbildung mit einem Curriculum, das zwei Zielen dient: Es soll zum einen eine systematische wissenschaftliche Grundlage für die hochspezialisierten Forschungsprojekte liefern, an denen die Studierenden arbeiten. Es soll aber auch ein breiteres Spektrum theoretischer Kenntnisse der Toxikologie bereitstellen, die die Absolventinnen und Absolventen des Graduiertenkollegs in die Lage versetzen, Arbeitsplätze in sehr unterschiedlichen Gebieten der toxikologischen Forschung und der Regulation in Universität, Behörden und Industrie auszufüllen. Das Konzept basiert auf den Erfahrungen früherer toxikologische Graduiertenkollegs Graduiertenkolleg „Toxikologie und Umwelthygiene“ und Graduiertenkolleg 738 „Molecular Mechanisms in Food Toxicology“ (internationales Graduiertenkolleg gemeinsam mit niederländischen Universitäten) - der Heinrich-Heine-Universität. Es besteht aus folgenden Komponenten: einem elektronischen Kurs oder einer Vorlesungsreihe zu Themen der allgemeinen und speziellen Toxikologie, Laborpraktika zu experimentellen Techniken, in denen einzelne Arbeitsgruppen des Kollegs besondere Erfahrungen haben, einem von den Studierenden selbst gestalteten Literaturseminar, ein- bis zweitägigen Symposien mit eingeladenen Sprechern aus dem In- und Ausland, Gastvorträgen, Exkursionen zu in der Region ansässigen Industrieunternehmen und Kongressbesuchen. Einmal jährlich wird ein zweitägiges Doktorandenkolloquium abgehalten, bei dem alle Studierenden über den Stand ihrer Arbeit berichten. Die Studierenden werden jeweils zwei Hochschullehrern zugeordnet, dem (der) Arbeitsgruppenleiter(in) und dem (der) Leiter(in) einer Gruppe mit verwandter Thematik; beide zusammen übernehmen die Betreuung und bewerten den Fortgang des Dissertationsprojektes. Eine besondere Komponente des Curriculums ist die Teilnahme an den Fortbildungskursen der Deutschen Gesellschaft für experimentelle und klinische Pharmakologie und Toxikologie (DGPT) oder ersatzweise an den entsprechenden Kursen des niederländischen Programms „Postgraduate Education in Toxicology“ (P.E.T), die auf die Erteilung der Bezeichnung „Fachtoxikologe/Fachtoxikologin DGPT“, eine bei allen potenziellen Arbeitgebern in Industrie und Behörden hochgeschätzte Qualifikation, hinzielen. In Deutschland und im Bereich der gesamten EU besteht ein Mangel an qualifizierten Fachkräften in der Toxikologie nicht nur auf dem Gebiet der Lebensmittelsicherheit, sondern in Anbetracht der 2007 in Kraft getretenen europäischen Chemikaliengesetzgebung auch in anderen Feldern der Toxikologie. Im Kontrast dazu ist nicht nur in Deutschland, sondern auch in einer Reihe anderer europäischer Länder die Zahl der Universitätsinstitute für Toxikologie im letzten Jahrzehnt sogar zurückgegangen. Die Existenz eines Graduiertenkollegs mit toxikologisch ausgerichtetem Curriculum ist geeignet, die Position des Faches in der Universitätslandschaft zu stärken und dem Mangel an qualifizierten Toxikologinnen und Toxikologen abzuhelfen. 


\title{
Überblick über die Teilprojekte
}

\section{Projekt 1: Interaktion von Flavonoiden mit der AhR-Signalkaskade: Effekte auf Absorption, Transport und Metabolismus von Fremdstoffen}

Projektleiter: Univ.-Prof. Dr. Josef Abel (IUF), PD Dr. Peter Roos (IfADo)

Stipendiaten: Hanno Bothe, Jeanette Niestroy

Der wichtigste Expositionspfad für viele giftige Stoffe, die in der Nahrung vorkommen können, ist der Darm. Über diesen Weg werden neben Naturstoffen auch zahlreiche Umweltchemikalien, beispielsweise verschiedene Benzpyrenderivate und Dioxine, enteral aufgenommen. Eine zentrale Bedeutung bei der Entfaltung der toxischen Wirkungen der genannten Fremdstoffe im Organismus hat der Arylhydrokarbon-Rezeptor (AhR), an den sie mit hoher Affinität binden können. Auch Flavonoide sind in der Lage, mit der AhRSignalkaskade zu interagieren. In diesem Projekt soll deshalb untersucht werden, welchen Einfluss eine Interaktion zwischen Flavonoiden und dem AhR-Signalweg auf die Aufnahme, den Transport und den Metabolismus von Fremdstoffen ausübt. Das Untersuchungsprogramm umfasst sowohl In-vitro- als auch In-vivo-Studien an gentechnisch veränderten Zellen und Versuchstieren. Aus den Daten können neue Ansatzpunkte für präventive Strategien zur Reduzierung der inneren Exposition mit Fremdstoffen gewonnen werden.

\section{Projekt 2: Absorption und Metabolismus von Flavonoiden aus der Nahrung: Mechanistische Untersuchungen}

\author{
Projektleiter: Univ.-Prof. Dr. Peter Proksch, PD Dr. Wim Wätjen \\ (beide Heinrich-Heine-Universität) \\ Stipendiat: Sven Ruhl
}

Diejenigen Flavonoide, die in der Nahrung am häufigsten vorkommen, sind nicht notwendigerweise auch diejenigen, die im Körper die größte Wirkung entfalten, sei es, dass sie nur eine geringe intrinsische Aktivität haben, unzureichend aus dem Darm resorbiert werden oder schnell eliminiert werden. Kenntnisse zur Bioverfügbarkeit und zum Metabolismus der Flavonoide sind deshalb unverzichtbar für die Identifikation derjenigen Einzelsubstanzen, denen die höchste biologische Aktivität zugewiesen werden kann. Die Ziele dieses Projektes sind es, (1.) die kinetischen Parameter und das Metabolitenprofil strukturell verwandter Flavonoide in verschiedenen Organen von Mäusen zu analysieren und sie mit organspezifischen prooxidativen, proapoptotischen und zytotoxischen Wirkungen in Zusammenhang zu setzen, (2.) in Transporter-defizienten Mäusen diejenigen Transportproteine zu identifizieren, die für die Aufnahme und Verteilung von Flavonoiden von Bedeutung sind, und (3.) die Ergebnisse aus den In-vivo-Studien mit Hilfe von Zellkultursystemen, die spezifische Transportproteine und Entgiftungsenzyme exprimieren, mechanistisch zu interpretieren. 


\title{
Projekt 3: Nahrungsmittelbestandteile als Modulatoren der humanen neuralen Entwicklung
}

\author{
Projektleiterin: \\ Dr. Ellen Fritsche (IUF) \\ Stipendiatin: \\ Kathrin Gassmann \\ Kollegiatinnen und Kollegiaten: Roland Pfeiffer, Timm Schreiber, Julia Tigges
}

Flavonoide sind sekundäre Pflanzeninhaltsstoffe mit polyphenolischer Struktur. Epidemiologische Studien weisen auf eine negative Korrelation zwischen der Flavonoidaufnahme des Menschen und dem Risiko für verschiedenste Krankheiten hin. Wegen dieser protektiven Eigenschaften werden Flavonoide in wachsendem Umfang als Nahrungsergänzungsmittel und als so genanntes „Functional Food“ angeboten. Dabei können Plasmakonzentrationen erreicht werden, die zehn- bis 100-fach über den Konzentrationen der durch die normale Nahrung aufgenommenen Flavonoide liegen. Die toxikologische Relevanz solch hoher Konzentrationen ist noch weitgehend unbekannt. Die biologischen Effekte der Flavonoide werden überwiegend ihren antioxidativen Eigenschaften zugeschrieben. Neuere Forschungsergebnisse weisen jedoch darauf hin, dass Flavonoide mit einer Vielzahl von Zellsignalwegen wie zum Beispiel mit dem Arylhydrocarbonrezeptor interagieren können. Die entwicklungsbiologische Bedeutung dieser Interaktionen ist noch unerforscht und adverse Effekte von Flavonoiden sind somit nicht auszuschließen. An einem In-vitro-Modell basierend auf der Kultivierung von menschlichen neuralen Progenitorzellen wird der Einfluss verschiedener Flavonoide auf wichtige neurale Entwicklungsprozesse wie Migration, Adhäsion, Differenzierung und Apoptose untersucht.

\section{Projekt 4: Testosteron-induzierte persistente Dysregulation des Leber-Transkriptoms}

Projektleiter: Univ.-Prof. Dr. Frank Wunderlich (Heinrich-Heine-Universität)

Stipendiat: Denis Delic

Der Missbrauch von Testosteron unter Sportlern, in der Bodybuilderszene sowie in der Tiermast ist beunruhigend. Denn Testosteron ist bekannt für seine immunsuppressiven Eigenschaften und führt zu erhöhter Suszeptibilität gegenüber einer Reihe von Infektionskrankheiten. Beispielsweise verlieren weibliche C57BL/6-Mäuse nach Testosteronbehandlung ihre Resistenz gegenüber Plasmodium chabaudi-Malaria, und dieser Verlust ist selbst zwölf Wochen nach Absetzen des Testosterons noch zu beobachten. Die Analyse des Leber-Transkriptoms mittels Affymetrix-DNA-Chips und quantitativer real time-PCR ergab nicht nur markante Kurz-, sondern auch Langzeitveränderungen nach Testosteronbehandlung. So kommt es bei weiblichen Mäusen zu einer Virilisierung der Genexpression, zu dauerhafter Repression von weibchenspezifischen Genen, zu einer Zunahme kanzerogener Marker-Gene und zu einer Leber-Pankreas Transdifferenzierung. Von den nukleären Rezeptoren ist vor allem die Expression des Vitamin-D-Rezeptors dauerhaft supprimiert. Derzeit wird dessen Rolle bei den Testosteron-induzierten Langzeitveränderungen untersucht. 


\title{
Projekt 5: Cadmiumeffekte auf Östrogenrezeptor-vermittelte Signalprozesse in Colonzellen und Bewertung der hormonalen Potenz in vivo
}

\author{
Projektleiter: Univ.-Prof. Dr. Gisela H. Degen (IfADo), PD Dr. Patrick Diel, (Deutsche \\ Sporthochschule Köln) \\ Stipendiatin: Nicola Höfer
}

\begin{abstract}
Das toxische Metall Cadmium ist eine Umweltkontaminante, die der Mensch über verschiedene Pfade, zum Beispiel mit Tabakrauch und mit der Nahrung, aufnimmt. Cadmium wird inzwischen als potenzieller endokriner Disruptor angesehen, denn es kann über Interaktion mit Estrogenrezeptoren auch hormonartige Wirkungen entfalten. Unsere ersten Befunde an Nagern zeigen allerdings, dass eine solche Wirkung maßgeblich vom Applikationspfad bestimmt wird: Nur bei intraperitonealer Gabe von Cadmiumchlorid sind östrogene Wirkungen in Zielorganen nachweisbar; bei oraler Gabe stehen wegen der geringen Bioverfügbarkeit des Metalloöstrogens lokale Effekte am Darm im Vordergrund. Da im Darm zwei Estrogenrezeptorsubtypen (alpha und beta) exprimiert werden, konzentrieren sich weitere Untersuchungen in vivo und an Zellkulturen nun auf Rezeptorvermittelte Wirkungen von Cadmium und die Aufklärung der zugrunde liegenden molekularen Mechanismen. Ziel dieser Studien ist eine für die toxikologische Bewertung nutzbare Charakterisierung des Wirkprofils von Cadmium.
\end{abstract}

\section{Projekt 6: Rolle von Flavonoiden aus der Nahrung bei der Aktivierung des Antioxidant Responsive Element (ARE) und der Induktion von Phase-II- Enzymen des Fremdstoffmetabolismus}

\author{
Projektleiterin: Univ.-Prof. Dr. Regine Kahl (Heinrich-Heine-Universität) \\ Stipendiatin: Ricarda Rohrig \\ Kollegiatin: Regine Lüpertz
}

Das Antioxidant Responsive Element (ARE) findet sich in der Promotorregion einer Batterie von Genen, die für die Entgiftungsenzyme des Phase-II-Stoffwechsels (zum Beispiel die NADPH-Chinon-Oxidoreduktase, die Glutathiontransferasen und die $\gamma$-Glutamylcysteinsynthetase) codieren. Diese Enzyme schützen die Zellen auch vor einem oxidativen Stress. Paradoxerweise leitet sich die Benennung des ARE von der Beobachtung ab, dass es durch synthetische phenolische Antioxidantien aktiviert werden kann; tatsächlich sind es aber prooxidative Stoffwechselprodukte dieser Antioxidantien, die die Aktivierung verursachen. Die in Lebensmitteln und Nahrungsergänzungsmitteln vorkommenden Flavonoide ähneln diesen synthetischen Antioxidantien in vieler Hinsicht, auch darin, dass sie prooxidative Wirkungen ausüben können: Die Hydrochinon- oder Catecholstruktur der potentesten Flavonoid-Antioxidantien bedingt auch ihre Neigung zum Redoxzyklus und zur Bildung reaktiver Sauerstoffspezies in der Zelle. Man darf auch nicht aus den Augen verlieren, dass Zellen auf oxidativen Stress mit einer koordinierten Adaptationsantwort reagieren und exogene Antioxidantien wie die Flavonoide mit dieser Adaptation interferieren können. Das Projekt beschäftigt sich mit der Frage, wie Flavonoide die Induktion von Phase-II-Enzymen im Darm beeinflussen und ob ihr Einfluss über eine Aktivierung des ARE vermittelt wird. 


\section{Projekt 7: Oxidativer Stress und die Wirkung von Antioxidantien in membranabhängigen Signaltransduktionsprozessen ausgelöst durch Nanopartikel in Epithelzellen}

Projektleiter: Dr. Klaus Unfried (IUF)

Stipendiatin: Henrike Peuschel

Kollegiat: Alexander Weissenberg

Die Interaktion von Nanopartikeln mit Epithelzellen löst eine Reihe von Ereignissen aus, die zu pathogenen Endpunkten wie Apoptose, Proliferation und Ausschüttung von proinflammatorischen Zytokinen führt. Der entscheidende Parameter für die Induktion dieser Zellreaktionen ist die Partikelgröße. In früheren Arbeiten der Gruppe konnte gezeigt werden, dass membranabhängige Signaltransduktionsprozesse unter Beteiligung der Membranrezeptoren EGF-R und $\beta_{1}$-Integrin die spezifische Reaktion auf Nanopartikel vermitteln. Die laufenden Arbeiten des Projekts befassen sich mit der Rolle von reaktiven Sauerstoffspezies (ROS), die durch Nanopartikel induziert werden können, an der Auslösung der Signalkaskaden. Das oxidative Potenzial verschiedener Nanopartikel sowie der jeweilige Einfluss auf Signalprozesse und Endpunkte werden durch ROS-Messungen und Versuche mit Antioxidantien untersucht. Weiterhin werden rezeptortragende Membranstrukturen wie Fokalkontakte und lipid rafts bezüglich ihrer Bedeutung für nanopartikelspezifische Effekte betrachtet. Methoden der Zellfraktionierung sowie Kinaseassays und spezifische Ausschaltung einzelner Signalproteine dienen dazu, die Interaktion von Membranrezeptoren und ihre Lokalisierung in den genannten Membranbereichen nachzuweisen.

\section{Projekt 8: Die Rolle des Ah-Rezeptors bei der Immunantwort von darmassoziiertem lymphoidem Gewebe: Ist Dioxin ein Inhibitor oder ein Mediator der oralen Immuntoleranz?}

Projektleiterin: Univ.-Prof. Dr. Charlotte Esser (IUF)

Stipendiatin: Stefanie Chmill

Kollegiatin: Stephanie Kadow

Der Darm ist einerseits gegenüber infektiösen Agentien exponiert, andererseits kommt er ständig mit harmlosen Nahrungsinhaltsstoffen in Berührung. Während gegenüber den Ersteren eine Immunantwort stattfinden muss, ist Toleranz - Immunosuppression - gegenüber den Letzteren lebenswichtig. Das Immunsystem hat Mechanismen zum Umgang mit diesem Paradox entwickelt. Allerdings sind die Vorgänge, die die empfindliche Balance zwischen der Auslösung der Immunantwort und der nicht-immunresponsiven Grundsituation gewährleisten, nicht völlig bekannt. Wenig Information liegt auch zu den Wirkungsmechanismen immuntoxischer Substanzen auf das Immunsystem des Darms vor. So ist erstaunlich wenig bekannt über die Effekte von 2,3,7,8-Tetrachlorodibenzo-p-dioxin (TCDD) auf sein Erstkontaktgewebe. Neben TCDD sind viele weitere Nahrungsinhaltsstoffe, zum Beispiel Flavonoide, Indole oder bakterielle Tryptophanabkömmlinge, Liganden des Transkriptionsfaktors Arylhydrocarbonrezeptor (AhR) und können so die AhRvermittelte Genmodulation im Darm aktivieren. In dem Projekt soll die Rolle des AhR auf das Gleichgewicht zwischen tolerogenen und inflammatorischen Prozessen im Darm 
bei der Einwirkung von Nahrungsinhaltsstoffen, die AhR-Liganden sind, untersucht werden. Die AhR-vermittelte Genexpression ist zell- und zellstatusspezifisch. Es ist deshalb zu erwarten, dass sich die Reaktionen von Immunzellen des Darms von denen peripherer Immunzellen unterscheiden. Untersuchungen zum Homöostasemechanismus im Immunsystem des Darms sind die Grundlage für die Vermeidung von Lebensmittelallergien und die Entwicklung oraler Impfstrategien.

\section{Projekt 9: Effekte von Nanopartikeln im Darm}

Projektleiter:

Dr. Roel Schins, Univ.-Prof. Dr. Irmgard Förster (beide IUF)

Stipendiatinnen: Kirsten Gerloff, Meike Winter

Kollegiatinnen und Kollegiaten: Theresa Koehler, Agnes Scherbat, Anton Wessels, Verena Wilhelmi

Ziel des Projektes ist es, herauszufinden, ob Nanopartikel (Partikel kleiner als $100 \mathrm{~nm}$ in einer Dimension), die als Additive oder Verunreinigungen in der Nahrung vorkommen, toxische und immunologische Effekte sowie Beeinträchtigungen der Genomintegrität im Magen-Darm-Trakt hervorrufen können. In zwei Teilprojekten werden mit Hilfe eines murinen Colitismodells und mittels verschiedener In-vitro-Systeme (Kulturen von Darmepithelzellen und inflammatorischen Zellen wie dendritischen Zellen (DC) und neutrophilen Granulozyten) relevante Nanopartikel untersucht. Hierfür werden $\mathrm{TiO}_{2}$ (Farbstoff) und $\mathrm{SiO}_{2}$ (Bindemittel) sowie $\mathrm{ZnO}$ und $\mathrm{MgO}$, die zum Beispiel aufgrund antimikrobieller Eigenschaften in der Nahrungsmittelverpackungsindustrie Verwendung finden, ausgewählt. Darüber hinaus wird Carbon Black als Modell für umweltrelevante Partikel mit Kohlenstoffkern verwendet, das nach Inhalation und anschließender Clearance in den Magen-Darm-Trakt gelangen kann. Einige der ausgewählten Nanopartikel führen zu einer erhöhten Zytotoxizität und Senkung des Glutathionspiegels in den Darmepithelzelllinien CaCo-2 und HCT-116. Mit Hilfe des fpg-modifizierten Kometassays konnte eine erhöhte Induktion von DNA-Strangbrüchen und oxidativen DNA-Schäden gezeigt werden, jedoch war die Höhe der Induktion partikelspezifisch. Ebenso kann es zu einer Aktivierung des oxidativen Bursts in neutrophilen Granulozyten nach Belastung mit spezifischen Partikeln kommen. Außerdem konnte im Mausmodell nach oraler Applikation von $\mathrm{TiO}_{2}$ eine Verbesserung der Symptome einer DSS-induzierten Colitis (insbesondere eine Reduktion der Entzündung) nachgewiesen werden. Inkubation von knochenmarksabgeleiteten DC mit $\mathrm{TiO}_{2}$ und $\mathrm{SiO}_{2}$ zeigte eine Verschiebung des Anteils unreifer zugunsten reifer DC, was auf eine erhöhte Induktion von Apoptose, speziell in unreifen Zellen, zurückzuführen sein könnte.

\section{Rückblick auf 18 Monate Graduiertenkolleg 1427}

Das Kolleg hat seine Arbeit im Jahr 2006 mit acht Stipendiatinnen und Stipendiaten und mit fünf Kollegiatinnen und Kollegiaten aufgenommen; zum Ende des Wintersemesters 2007/2008 hat sich die Zahl auf elf Stipendiatinnen und Stipendiaten und zehn Kollegiatinnen und Kollegiaten mit Hochschulabschlüssen in Biologie, Biochemie, Chemie und Ökotrophologie erhöht. Sieben von ihnen haben ihren Abschluss an der Heinrich-Heine- 
Universität erworben, die übrigen an anderen deutschen und österreichischen Hochschulen. Gegen Ende des Jahres 2007 wurden mit allen Stipendiatinnen und Stipendiaten und mit einem Teil der Kollegiatinnen und Kollegiaten Einzelgespräche über den Status und die Aussichten der Dissertationsprojekte geführt, bei denen die beiden Betreuer und die Sprecher des Kollegs anwesend waren. Alle diese Statusgespräche verliefen positiv. Die Studierenden nahmen die Möglichkeit zum Besuch von Kongressen, auf denen sie ihre Arbeiten vorstellten, und Fortbildungskursen zahlreich wahr. Vom Graduiertenkolleg selbst wurde im Oktober 2007 in den Räumen der Heinrich-Heine-Universität ein Symposium mit dem Titel „Food, Gut and the Immune System: a Challenge in Environmental Medicine“" mit Sprechern aus deutschen Hochschulen und Hochschulen in den USA, Großbritannien und Spanien veranstaltet; ein zweites Symposium, diesmal mit dem Titel „Nutrition and Health: Intestinal Effects of Food Constituents“, wird im April 2008 ebenfalls unter internationaler Beteiligung in Düsseldorf stattfinden. Es ist bereits eine Reihe von Publikationen unter Beteiligung von Studierenden des Graduiertenkollegs in international renommierten Zeitschriften mit Peer-Review-System erschienen. Man kann also davon ausgehen, dass das Graduiertenkolleg 1427 in Forschung und Lehre auf gutem Wege ist. 